\title{
Comprimento do Colo Uterino de Gestantes com Rotura Prematura Pré-Termo de Membranas Avaliado pela Ultra-sonografia Transvaginal
}

Length of the Uterine Cervix by Transvaginal Ultrasonography in Pregnant Women with Preterm Rupture of Membranes

Patricia Spara, Francisco Mauad Filho, Antonio Gadelha da Costa, Adilson Cunha Ferreira, Reginaldo Antônio de Oliveira Freitas-Júnior, Aderson Tadeu Berezowski

\section{RESUMO}

Objetivo: avaliar o comprimento do colo uterino, por meio da ultra-sonografia transvaginal, em gestantes com rotura prematura pré-termo de membranas.

Pacientes e Métodos: o grupo de estudo (Ge) foi constituído por 26 grávidas entre a $24^{a}$ e a $36^{a}$ semana de gestação e o grupo controle (Gc) por 49 pacientes clinicamente normais, na mesma idade gestacional. As pacientes foram avaliadas entre a $24^{a}$ e $28^{a}, 28^{a}$ e $32^{a}, 32^{a}$ e $36^{a}$ semana de gestação, sendo subdivididas nos subgrupos Ge24-28, Ge28-32, Ge32-36 e Gc24-28, Gc28-32, Gc32-36, conforme pertencessem ao grupo de estudo e controle, respectivamente. O comprimento do colo uterino foi obtido por meio da ultra-sonografia transvaginal, em vista sagital, realizando-se a medida linear da distância entre os orificios cervicais interno e externo.

Resultados: observamos diferenças significantes das medidas do comprimento do colo uterino entre Ge24-28 e Gc24-28, cujos valores foram 24,3 e 33,0 mm, respectivamente ( $p=0,04)$, como também entre Ge32-36 e Gc32-36, cujos valores foram, respectivamente, 20,1 e 28,0 $m m(p=0,005)$. Os períodos de latência dos grupos Ge24-28, Ge28-32 e Ge32-36 foram, respectivamente, sete, cinco e três dias, apresentando correlação positiva com o comprimento do colo uterino $(r=0,66)$ e negativa com a idade gestacional $(r=-0,27)$.

Conclusão: o comprimento do colo uterino variou de acordo com a idade gestacional em que a rotura prematura pré-termo das membranas foi detectada, sendo menor no grupo de estudo que no grupo controle entre a $24^{a}$ e a $28^{a}$ e entre a $32^{a}$ e a $36^{a}$ semana. Além disso, demonstrou-se que quanto menor o comprimento do colo, menor é o tempo de latência, e que houve diminuição no período de latência à medida que aumentou a idade gestacional em que se deu a rotura.

PALAVRAS-CHAVE: Prematuridade. Colo uterino. Rotura prematura de membranes. Periodo de latência.

\section{Introdução}

A prematuridade continua sendo a principal causa de mortalidade perinatal, sendo que a rotura prematura de membranas amnióticas (RPM) é responsável por aproximadamente 30\% de

Departamento de Ginecologia e Obstetrícia da Faculdade de Medicina de Ribeirão Preto da Universidade de São Paulo. Correspondência:

Patricia Spara

Rua Iguape, 603/53 - Jardim Paulista

14090-090 - Ribeirão Preto - SP

e-mail: pspara@ig.com.br todos os nascimentos pré-termo. A incidência de RPM atinge, de maneira geral, $10 \%$ de todas as gestações. Quando a rotura prematura pré-termo de membranas (RPPM) é pesquisada separadamente, verifica-se que sua incidência é muito menor que a RPM próxima ao termo, variando de 1 a $3 \%$ na população obstétrica geral ${ }^{1,2}$.

$\mathrm{O}$ intervalo entre a rotura das membranas e o início do trabalho de parto é denominado período de latência e varia, na ausência de infecção intra-uterina, em razão inversa à idade gestacional em que ocorre a rotura ${ }^{3,4}$.

A ultra-sonografia transvaginal tem se mostrado ser o método de eleição para a investigação 
das modificações cervicais na gravidez, principalmente no que diz respeito à predição de parto prétermo ${ }^{5,6}$. Em nosso meio, Freitas-Júnior et al. ${ }^{7}$ realizaram estudo longitudinal que avaliou o colo uterino, por meio da ultra-sonografia transvaginal de 49 grávidas, a partir da $12^{\mathrm{a}}$ semana de gestação até o termo, demonstrando diminuição crescente do comprimento do colo uterino ao longo da gestação, sendo significante a partir da $20^{\mathrm{a}}$ semana e, mais expressivo, a partir da $28^{\mathrm{a}}$ semana.

Os estudos que dizem respeito à avaliação ultra-sonográfica cervical em pacientes com rotura prematura pré-termo de membranas amnióticas são escassos ${ }^{8,9}$. Gire et al. ${ }^{9}$, avaliando 101 gestantes com RPPM, demonstraram que o tempo médio entre a rotura e o parto foi de 48 horas. Esses autores observaram que um ponto de corte de $20 \mathrm{~mm}$ pode predizer um risco de parto precoce, com período de latência de 59,4 $\pm 159,9$ horas.

É conhecida e estabelecida a tendência obstétrica atual ao manejo conservador da RPPM. Sabe-se que a manutenção do feto in utero, tem sido a preferência da obstetrícia moderna. Assim sendo, a verificação de valores do comprimento do colo em gestantes acometidas por RPPM, objetivo desse trabalho, relacionando-os com variáveis como idade gestacional e período de latência, poderá contribuir para o estabelecimento de parâmetros nessas condições clínicas.

\section{Pacientes e Métodos}

Foi realizado estudo prospectivo de mensuração do comprimento cervical de 31 pacientes com RPPM, com idade gestacional entre 24 e 36 semanas (grupo de estudo), comparando-os com os valores obtidos em 49 grávidas normais do ponto de vista clínico e laboratorial, com idade gestacional semelhante (grupo controle). Foram excluídas cinco grávidas do grupo de estudo por apresentaram cultura endocervical positiva para Streptococcus agalactiae, restando, neste grupo, 26 pacientes. As pacientes foram selecionadas de forma aleatória de acordo com os critérios de inclusão e exclusão, durante o período de abril de 2000 a janeiro de 2003.

Foram considerados critérios de exclusão do grupo controle: gestações múltiplas, doenças próprias ou intercorrentes à gestação estudada, ameaça de parto pré-termo, gestações ultimadas antes da $37^{\mathrm{a}}$ semana, rotura prematura de membranas, restrição de crescimento intra-uterino, doença ou malformação fetal, malformação uterina, antecedentes de cirurgia prévia no colo uterino (conização, amputação, cerclagem), antecedentes de amniorrexe prematura ou partos prematuros anteriores.

Para o grupo de estudo consideraram-se cri- térios de exclusão os seguintes: gestações múltiplas, doenças próprias ou intercorrentes à gestação estudada, malformação fetal, malformação uterina, antecedentes de cirurgia prévia no colo uterino (conização, amputação, cerclagem), diagnóstico de RPPM em tempo superior a 24 horas do início da perda líquida vaginal referida pela paciente, tocólise ou uso de corticóides previamente à internação. Todas as pacientes assinaram o termo de consentimento informado.

A idade gestacional das pacientes dos dois grupos foi estabelecida a partir da data da última menstruação e confirmada por exame ultrasonográfico realizado até a décima segunda semana de gestação, ou por, pelo menos, dois exames ultra-sonográficos concordantes realizados entre 12 e 20 semanas de gestação.

As medidas do comprimento do colo uterino do grupo controle foram realizadas com as idades gestacionais compreendidas entre 24 e 28 semanas (Gc24-28), 28 e 1/7 e 32 semanas (Gc28-32) e 32 e $1 / 7$ e 36 semanas (Gc32-36). Todos estes subgrupos somados consistiam de 49 pacientes. Para o grupo de estudo, utilizaram-se as idades gestacionais compreendidas entre 24 e 28 semanas (Ge24-28), 28 e 1/7 e 32 semanas (Ge28-32) e 32 e 1/7 e 36 semanas (Ge32-36). O subgrupo (Ge2428) foi formado por cinco pacientes, o subgrupo (Ge28-32) por 14 pacientes e o subgrupo (Ge32-36) por sete pacientes. Desta forma, as medidas do colo de Gc24-28, Gc28-32 e Gc32-36 foram cotejadas com as de Ge24-28, Ge28-32 e Ge32-36.

Procurou-se, ainda, estabelecer o período de latência, relacionando-o com a idade gestacional e com o comprimento do colo uterino das gestantes do grupo de estudo. O período de latência foi definido como o intervalo, em dias, entre a rotura das membranas amnióticas e o desencadeamento do trabalho de parto.

Todos os exames do grupo controle e de estudo foram realizados por um único observador, utilizando-se sempre o mesmo aparelho ultrasonográfico.

As pacientes foram colocadas em posição ginecológica, em decúbito dorsal, com as pernas abduzidas, após esvaziamento vesical completo. $\mathrm{O}$ transdutor ultra-sonográfico transvaginal, de 5 a $9 \mathrm{MHz}$, foi coberto totalmente com preservativo estéril não lubrificado, contendo pequena quantidade de gel transmissor em seu reservatório e, para facilitar a inserção do transdutor no intróito vaginal, utilizando-se vaselina estéril sobre sua extremidade para lubrificação. O transdutor foi introduzido em direção ao fórnix vaginal anterior, mantido no terço externo do canal vaginal, de forma a se obter vista sagital do colo uterino, sem atingir a cérvice. Identificavam-se os orifícios interno e externo, canal cervical e mucosa 
endocervical, esta última utilizada para definição precisa do nível do orifício interno e construção da imagem do canal cervical em toda sua extensão. O campo visual foi ampliado, de tal forma que o colo uterino ocupasse cerca de $75 \%$ da imagem estudada. Procedeu-se à medida linear da distância entre o orifício interno e o externo, efetuandose três imagens com uma medida em cada imagem, durante um período mínimo de 3 minutos. Registrou-se a mais curta medida do colo uterino. Todas as medidas foram realizadas na ausência de alterações dinâmicas do colo uterino.

A análise estatística foi feita por meio do coeficiente de correlação de Pearson, análise de variância e teste não-paramétrico de MannWhitney. Em todas as análises o nível de significância foi $\mathrm{p}<0,05$.

As variáveis descritivas foram idade gestacional no momento da rotura, tipo de parto, indicação de cesárea, quando ocorrida, e internação em centro de tratamento intensivo neonatal (CTIN), comprimento do colo uterino e período de latência.

O cálculo do tamanho amostral (n) foi obtido utilizando-se a fórmula $n=Z^{2} \times(p \times q) / i^{2}$, onde $Z$ significa o valor do nivel de significância desejado, p significa a proporção estimada para a ocorrência do evento em estudo (no caso, a paciente acometida por RPPM), q significa o complementar de p, e i significa a variabilidade aceitável da referida proporção. Assim sendo, foi estabelecido nível de significância de 5\%, determinando-se um $Z$ de 1,96. Consideramos incidência de $3 \%$ de RPPM ${ }^{1,2}$. Portanto, aceitou-se 0,03 como o valor aproximado de p, o que determinou valor de q de $97 \%(0,97)$. Finalmente, foi determinado que seria aceito valor de i de até $10 \%(0,1)$. Dessa forma, obtivemos um $\mathrm{n}$ de 11,2 , correspondendo a 12 pacientes.

$\mathrm{O}$ estudo foi analisado e aprovado pelo Comitê de Ética em Pesquisa do Hospital das Clínicas da Faculdade de Medicina de Ribeirão Preto da Universidade de São Paulo.

\section{Resultados}

A idade das pacientes variou de 14 a 43 anos. Com relação à idade gestacional no momento da rotura, prevaleceu a de 31 semanas, perfazendo $26,6 \%$ da amostra, sendo que a média foi de 30,5 $\pm 2,8$ semanas.

Quando se avaliaram as gestantes em relação ao tipo de parto, observou-se predomínio do vaginal espontâneo, perfazendo $65,4 \%$ dos casos. A cesárea ocorreu em $34,6 \%$ das grávidas, sendo o sofrimento fetal agudo $(33,4 \%)$, apresentação pélvica $(22,2 \%)$ e situação transversa $(22,2 \%)$ as principais indicações.
Por se tratar de gestações com fetos prétermo, o número de internações dos recém-nascidos no CTIN foi significativo, perfazendo $61,5 \%$ de todos os casos. A principal causa de internação desses recém-nascidos no CTIN foi desconforto respiratório precoce (50\%), seguido de prematuridade extrema $(18,8 \%)$, prematuridade extrema associada a desconforto respiratório precoce $(12,6 \%)$, peso ao nascimento abaixo de 1.500 gramas $(6,2 \%)$, apnéia $(6,2 \%)$ e choque hipovolêmico $(6,2 \%)$. Todos receberam alta hospitalar em boas condições. As grávidas incluídas no estudo não apresentaram infecção.

O comprimento do colo uterino do grupo controle mostrou valores progressivamente decrescentes ao longo da gestação. No grupo de estudo, essa medida variou de acordo com a idade gestacional em que foi detectada a RPPM. Assim sendo, o percentil 50 da medida do colo uterino entre a $24^{\mathrm{a}}$ e a $28^{\mathrm{a}}$ semana de gestação (Ge24-28) foi 24,30 $\mathrm{mm}$, entre a $28^{\mathrm{a}}$ e a $32^{\mathrm{a}}$ (Ge28-32), 31,60 mm, e entre a $32^{\mathrm{a}}$ e a $36^{\mathrm{a}}$ (Ge32-36), 20,0 mm (Tabela 1 ).

Tabela 1 - Percentis do comprimento do colo uterino das gestantes dos grupos de estudo e controle nas semanas gestacionais avaliadas.

\begin{tabular}{lccccc}
\hline Grupos & p5 & p25 & p50 & p75 & p95 \\
\hline Ge24-28* & 17,6 & 18,4 & 24,3 & 34,2 & 40,8 \\
Gc24-28 & 23,9 & 30,3 & 33,0 & 36,0 & 44,9 \\
Ge28-32** & 10,1 & 19,4 & 31,6 & 35,9 & 42,3 \\
Gc28-32 & 19,1 & 27,3 & 29,5 & 34,3 & 40,1 \\
Ge32-36** & 16,1 & 16,5 & 20,1 & 24,1 & 31,6 \\
Gc32-36 & 16,7 & 23,3 & 28,0 & 30,0 & 37,0
\end{tabular}

Dados apresentados em $\mathrm{mm}$ por idade gestacional.

Ge: grupo de estudo; Gc: grupo controle.

Os subgrupos do Gc compreendem no total 96 pacientes.

Os subgrupos Ge24-28, Ge28-32 e Ge32-36 compreendem, respectivamente, 5, 14 e 7 pacientes.

*Comparou-se Ge24-28 com Gc24-28, Mann-Whitney, $p=0,04$.

**Comparou-se Ge28-32 com Gc28-32, Mann-Whitney, $p=0,86$

${ }^{\star * *}$ Comparou-se Ge32-36 com Gc32-36, Mann-Whitney, $p=0,005$

Comparando-se os valores do colo uterino de gestantes com RPPM com os das gestantes normais, observaram-se diferenças significantes das medidas do comprimento do colo uterino entre Ge24-28 e Gc24-28, cujos valores foram 24,3 e 33,0 $\mathrm{mm}$, respectivamente (Mann-Whitney, $\mathrm{p}=0,04$; Tabela 1), como também entre Ge32-36 e Gc3236 , cujos valores foram, respectivamente, 20,1 e 28,0 mm (Mann-Whitney, p=0,005; Tabela 1). Não se observou significância estatística, no entanto, entre as pacientes de 28 a 32 semanas (MannWhitney, $p=0,86$; Tabela 1).

A relação entre a idade gestacional e o período de latência não foi significante (ANOVA p=0,18), entretanto o período de latência foi superior nas 
grávidas que apresentaram maior comprimento do colo uterino e inferior naquelas com menor comprimento do colo uterino (ANOVA, p<0,001, coeficiente de correlação de Pearson = 0,66; Figura 1).

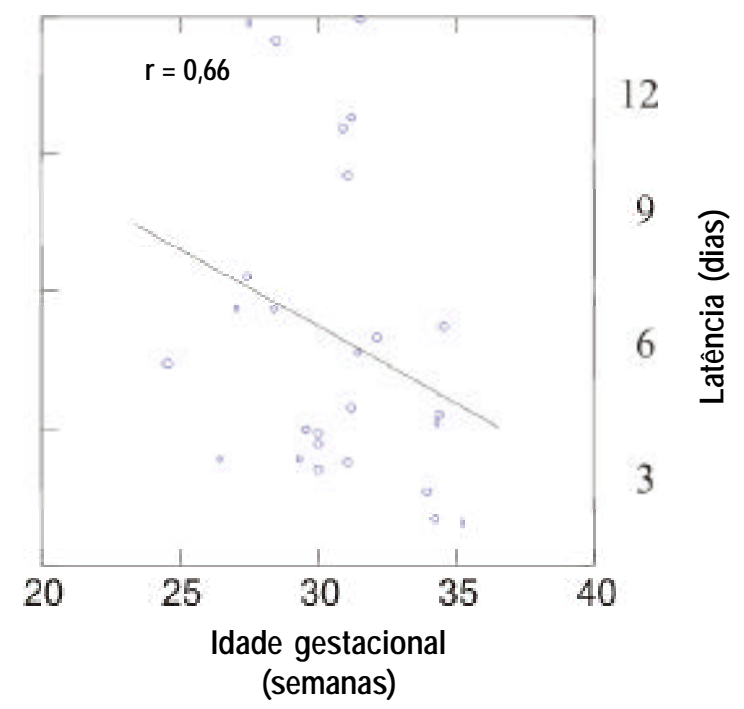

Figura 1 - Relação entre o período de latência e o comprimento do colo uterino em pacientes com rotura prematura pré-termo de membranas. Coeficiente de correlação de Pearson $=0,66$. Equação de regressão linear: $-2656,96+423,68 \times$ comprimento do colo uterino.

\section{Discussão}

Em pacientes com rotura das membranas amnióticas, alguns autores questionaram o exame do colo pela ultra-sonografia transvaginal, pelo fato de que o transdutor ultra-sônico poderia constituir fator de risco para a ascensão de germes à cavidade uterina e, portanto aumentar o risco de infecção intra-amniótica ${ }^{10,11}$. O exame digital em RPPM, para Lewis et al. ${ }^{12}$, diminui o período de latência, efeito provavelmente semelhante ao de um transdutor vaginal. Carlan et al. ${ }^{8}$ estudaram gestantes com RPPM entre a $24^{\mathrm{a}}$ e a $34^{\mathrm{a}}$ semana de gestação. Em um grupo de 45 mulheres utilizaram a ultra-sonografia endovaginal semanal, em outro constituído de 47 gestantes, não utilizaram. Observaram que corioamnionite, endometrite e infecção neonatal não foram diferentes entre os grupos. Encontraram, também, período de latência de 9,8 dias no grupo sem ultra-som e de 11,7 dias no grupo com ultrasom, sem diferença significante entre eles.

Os cuidados técnicos para a realização do exame pela ultra-sonografia podem eliminar a possibilidade de penetração do canal cervical, o que no presente estudo foi rigorosamente obedecido. Em 5 das 31 gestantes estudadas, encontrou-se a presença do Streptococcus agalactie detectada em cultura de secreção endocervical coletada previamente ao exame ecográfico. Dos 26 recém-nascidos, $61,5 \%$ foram internadas no centro de terapia intensiva neonatal (CTIN), e nenhum apresentou qualquer sinal de infecção neonatal. Os principais diagnósticos de CTIN foram desconforto respiratório precoce (50\%), prematuridade extrema $(18,8 \%)$, e a associação dessas duas entidades clínicas $(12,6 \%)$. Para Carlan et al. ${ }^{8}$, a ultra-sonografia endovaginal pode ser realizada semanalmente sem aumento de infecções maternas ou neonatais, ou decréscimo no período de latência. Gire et al. ${ }^{9}$ sugerem que o uso do ultra-som transvaginal para a medida do comprimento do colo uterino, em casos de RPPM, pode predizer precocemente um nascimento pré-termo, mas não antecipa o risco de corioamnionite ou sepse neonatal. Estes autores encontraram taxa de cesárea de $36 \%$ em seu material, semelhante ao observado no grupo com RPPM, 34,6\%.

A medida do comprimento do colo uterino tem atualmente papel destacado na predição de trabalho de parto pré-termo. Em nosso meio estudo longitudinal foi realizado, mostrando diminuição crescente do comprimento do colo uterino ao longo da gestação. A partir da $20^{\mathrm{a}}$ semana esse decréscimo foi significativo, sendo mais expressivo a partir da $28^{\mathrm{a}}$ semana ${ }^{7}$.

Quando foram comparadas as medidas do colo uterino entre as gestantes dos grupos controle e de estudo, nas mesmas faixas de idade gestacional, houve diferença significante entre os dois grupos, nas idades gestacionais precoces (24 e 28 semanas) e tardias (32 e 36 semanas). Era de se esperar que a diferença ocorresse ao longo de todo o período estudado. Entretanto, alguns fatores podem ter interferido, tais como tamanho amostral, dispersão significativa dos valores encontrados e modelo de estudo proposto. No entanto, não deixam dúvidas que o comprimento do colo uterino em grávidas com RPPM é menor que em grávidas normais, levandose em consideração a significância estatística encontrada entre os dois grupos na maioria das semanas gestacionais avaliadas (v. Tabela 1).

No presente estudo o período de latência nas diversas idades gestacionais avaliadas foi superior nas grávidas com maior comprimento do colo uterino e inferior nas grávidas com menor colo uterino ( $v$. Figura 1). Nossos dados estão de acordo com os de Gire et al. ${ }^{9}$, que relataram haver correlação significante entre período de latência e comprimento do colo uterino em grávidas com RPPM. Esses dados são relevantes, tendo em vista a relação existente entre o comprimento do colo uterino e parto pré-termo. Colos uterinos menores estão relacionados com parto pré-termo ${ }^{13}$, cujo maior risco foi encontrado 
em grávidas com colo uterino menor que $20 \mathrm{~mm}^{9}$. Da mesma forma, a avaliação ultra-sonográfica em gestações complicadas por RPPM melhora a predição do intervalo entre a admissão e o parto ${ }^{14}$.

Concluímos que o comprimento do colo uterino varia de acordo com a idade gestacional em que a rotura das membranas é detectada, sendo, entre a $24^{\mathrm{a}}$ e a $28^{\mathrm{a}}$ e entre a $32^{\mathrm{a}}$ e a $36^{\mathrm{a}}$, menor em pacientes com rotura prematura pré-termo de membranas que em pacientes normais. Quanto menor o comprimento do colo uterino, menor o período de latência.

\section{ABSTRACT}

Purpose: to assess the length of the uterine cervix by transvaginal ultrasonography in pregnant women with preterm premature rupture of membranes.

Methods: the study group (Ge) consisted of 26 pregnant women with gestational age between 24 and 36 weeks and the control group $(G c)$ of 49 clinically normal patients at the same gestational age. The patients were evaluated between the 24th to 28th, 28th to 32th and 32th to 36th weeks. The groups were divided into subgroups Ge24-28, Ge28-32, Ge32-36 and Gc24-28, Gc28-32, Gc32-36, according to the study or control group. The cervix length was measured by transvaginal ultrasonography as the linear distance between the internal and external cervical os. Results: we observed significant differences in cervix length between Ge24-28 and Gc24-28 groups whose values were, respectively, 24.3 and $33.0 \mathrm{~mm}(p=0.04)$, and between Ge3236 and $G c 32-36,20.1$ and $28.0 \mathrm{~mm}$, respectively $(p=0.005)$. The latency periods of Ge24-28, Ge28-32 and Ge32-36 were, respectively, seven, five and three days, showing a positive correlation with cervix length $(r=0.66)$ and a negative correlation with gestational age $(r=-0.27)$.

Conclusions: the length of the uterine cervix varied with the gestational age when premature preterm rupture of the membranes was detected, with the length being shorter in the study group than in the control group betweeen the 24th and 28th and 32th and 36th weeks. In addition, it was demonstrated that, the shorter the cervix length, the shorter the latency time, with a reduction in the latency period with increasing gestational age at the time of rupture.

KEYWORDS: Prematurity, Uterine cervix, Premature rupture of membranes, Latency period.

\section{Referências}

1. Kaltreider DF, Kohl S. Epidemiology of preterm delivery. Clin Obstet Gynecol 1980; 23:17- 31.

2. Tucker JM, Goldenberg RL, Davis RO, Copper RL, Winkler CL, Hauth JC. Etiologies of preterm birth in an indigent population: is prevention a logical expectation? Obstet Gynecol 1991; 77:343-7.

3. Garite TJ, Freeman RK, Linzey EM, Braly PS, Dorchester WL. Prospective randomized study of corticosteroids in the management of premature rupture of the membranes and the premature gestation. Am J Obstet Gynecol 1981; 141:508-15.

4. Taylor J, Garite TJ. Premature rupture of membranes before fetal viability. Obstet Gynecol 1984; 64:615-20.

5. Kurtzman JT, Goldsmith LJ, Gall SA, Spinnato JA. Transvaginal versus transperineal ultrasonography: a blinded comparison in the assessment of cervical length at midgestation. Am J Obstet Gynecol 1998; 179:852-7.

6. Owen J, Neely C, Northen A. Transperineal versus endovaginal ultrasonographic examination of the cervix in the midtrimester: a blinded comparison. Am J Obstet Gynecol 1999; 181:780-3.

7. Freitas-Júnior RAO, Mauad-Filho F, Duarte G, Ferreira AC, Freitas AKMSO, Azevedo GD. Evolução do comprimento cervical uterino na gestação, avaliado pela ultra-sonografia transvaginal. Rev Bras Ginecol Obstet 2003; 25:115-21.

8. Carlan SJ, Richmond LB, O'Brien WF. Randomized trial of endovaginal ultrasound in preterm premature rupture of membranes. Obstet Gynecol 1997; 89:458-61.

9. Gire C, Faggianelli P, Nicaise C, et al. Ultrasonographic evaluation of cervical length in pregnancies complicated by preterm premature rupture of membranes. Ultrasound Obstet Gynecol 2002; 19:565-9.

10.Sakamoto H, Takagi K, Masaoka N, et al. Clinical application of the perineal scan: prepartum screening for cord presentation. Am J Obstet Gynecol 1986; 155:1041-3.

11.Benacerraf BR. Examination of the second-trimester fetus with severe oligohydramnios using transvaginal scanning. Obstet Gynecol 1990; 75:491-3.

12.Lewis DF, Major CA, Towers CV, Asrat T, Harding JA, Garite TJ. Effects of digital examinations on latency period in preterm premature rupture of membranes. Obstet Gynecol 1992; 80:630-4.

13. Odibo AO, Talucci M, Berghella V. Prediction of preterm premature rupture of membranes by transvaginal ultrasound features and risk factors in a high-risk population. Ultrasound Obstet Gynecol 2002; 20:245-51.

14.Rizzo G, Capponi A, Angelini E, Vlachopoulou A, Grassi C, Romanini C. The value of transvaginal ultrasonographic examination of the uterine cervix in predicting preterm delivery in patients with preterm premature rupture of membranes. Ultrasound Obstet Gynecol 1998; 11:23-9.

Recebido em: 23/7/2003 Aceito com modificações em: 21/1/2004 